
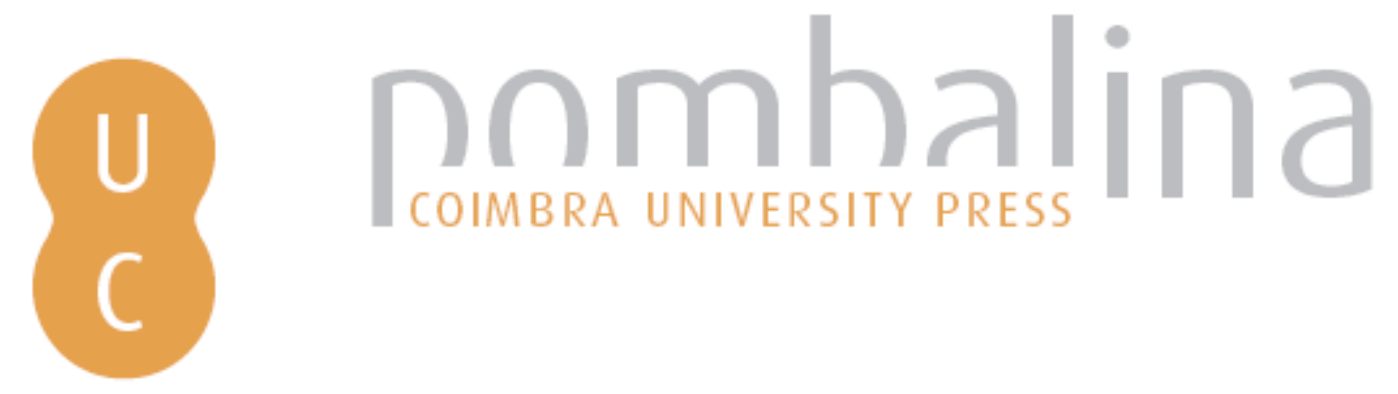

O desafio da divulgação de banco de dados geográficos por meio de SIG Web: o Parque Natural Municipal do Curió (Município de Paracambi - RJ, Brasil)

Autor(es): $\quad$ Costa, Vivian Castilho da; Paiva, Davyd Souza de

Publicado por: Imprensa da Universidade de Coimbra

URL

persistente: URI:http://hdl.handle.net/10316.2/37077

DOI: $\quad$ DOI:http://dx.doi.org/10.14195/978-989-26-0983-6_24

Accessed : $\quad$ 26-Apr-2023 10:38:32

A navegação consulta e descarregamento dos títulos inseridos nas Bibliotecas Digitais UC Digitalis, UC Pombalina e UC Impactum, pressupõem a aceitação plena e sem reservas dos Termos e Condições de Uso destas Bibliotecas Digitais, disponíveis em https://digitalis.uc.pt/pt-pt/termos.

Conforme exposto nos referidos Termos e Condições de Uso, o descarregamento de títulos de acesso restrito requer uma licença válida de autorização devendo o utilizador aceder ao(s) documento(s) a partir de um endereço de IP da instituição detentora da supramencionada licença.

Ao utilizador é apenas permitido o descarregamento para uso pessoal, pelo que o emprego do(s) título(s) descarregado(s) para outro fim, designadamente comercial, carece de autorização do respetivo autor ou editor da obra.

Na medida em que todas as obras da UC Digitalis se encontram protegidas pelo Código do Direito de Autor e Direitos Conexos e demais legislação aplicável, toda a cópia, parcial ou total, deste documento, nos casos em que é legalmente admitida, deverá conter ou fazer-se acompanhar por este aviso.

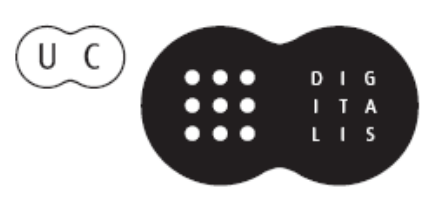




\section{$\forall$ \\ TAS DAS I JORNADAS LUSÓFONAS DE CIÊNCIAS E TECNOLOGIAS DE INFORMAÇÃO GEOGRÁFICA}

Editores

José Gomes dos Santos

Cidália Fonte

Rui Ferreira de Figueiredo

Alberto Cardoso

Gil Gonçalves

José Paulo Almeida

Sara Baptista 


\title{
ARTIGO 24
}

\section{O DESAFIO DA DiVULGaÇão de BANCO DE DADOS GEOGRÁficos POR MEIO DE SIG WEB: O PARQUE NATURAL MUNICIPAL DO CURIÓ (MUNiCípio DE PARACAMBI - RJ, BRASIL)}

COSTA, Vivian Castilho da ${ }^{1}$ \& PAIVA, Davyd Souza de 2

\begin{abstract}
Departamento de Geografia Física, Instituto de Geografia da; Universidade do Estado do Rio de Janeiro (Brasil); Laboratório de Geoprocessamento - LAGEPRO (Brasil); Rua São Francisco Xavier, 523, sala 4002, bloco B, Maracanã; CEP: 20550-013, Rio de Janeiro, RJ, Brasil; Tel: +55(21) 23340788; email: ${ }^{1}$ vivianuerj@ gmail.com \&22avydsp@gmail.com
\end{abstract}

\section{RESUMO}

A divulgação de mapas interativos na internet é primordial, sendo o objetivo principal deste trabalho que, ao utilizar o SIG Web ou Web GIS denominado i3Geo, construiu o Atlas Digital Ecoturístico para o Parque Natural Municipal do Curió (PNMC), área protegida localizada no município de Paracambi-RJ. Assim, usuários da Web podem utilizar o Atlas para simples visualização, até complexas análises ambientais. Como metodologia, a aquisição e o tratamento (por SIG livre) da base de dados georreferenciadas foi primordial à construção de MapFiles, ajustes internos e uso de sistema de administração da plataforma i3Geo. Como resultados finais, foi criada uma home-page e realizados testes em ambiente cliente-servidor da SERAD-SR2-UERJ e da Prefeitura Municipal de Paracambi, a fim de simular a consulta ao Atlas na Web.

\section{PALAVRAS-CHAVE}

SIG Web, Geoprocessamento, i3GEO, Atlas digital, Ecoturismo. 
THE CHALLENGE OF DisSEMINATING GEOGRAPHICAL DATA-

BASE THROUGH WEB GIS: THE MUNICIPAL NATURAL PARK OF CURIÓ (COUNTY PARACAMBI - RJ, BRAZIL)

\begin{abstract}
The dissemination of interactive maps on the Internet is prime, with the main objective of this work, that, by using the Web GIS called i3Geo, built a Digital Atlas of Ecotourism of the Municipal and Natural Park of Curió (PNMC), located in the municipality of Paracambi - RJ. Thus, Web users can use the maps of the Atlas for simple visualization to complex environmental analyzes. As methodology, the acquisition and processing (by using free GIS) georeferenced base data was primordial for building MapFiles, internal settings and using the administration system platform called i3Geo. As final results, home page was created and performed tests on client-server network installed in SERAD-SR2-UERJ and Prefecture of Paracambi City environment in order to simulate the query of Curió Atlas in Web.
\end{abstract}

\title{
KEYWORDS
}

Web GIS, Geoprocessing, i3Geo, Digital Atlas, Ecotourism.

\section{INTRODUÇÃO}

A emergência da questão ambiental, nos últimos anos, trouxe à tona a importância da preservação/conservação ambiental como peça fundamental para garantir a manutenção dos recursos naturais e dos serviços ambientais essenciais para a sociedade. A principal forma encontrada pelo Brasil para preservar áreas naturais foi a criação de Unidades de Conservação, que além de protegerem áreas naturais também se destinam à atividades recreativas e ecoturísticas. Mas como informar melhor a sociedade civil acerca do valor e da importância das áreas protegidas? Uma das formas é com mapas interativos na Web. Tais mapas temáticos servirão ao usuário leigo que deseja saber quais atrativos naturais tem em seu interior para a prática de ecoturismo, além de auxiliar os gestores da Unidade de Conservação no processo de gestão participativa e no manejo de seus recursos naturais.

É dentro deste contexto que se insere o presente trabalho, onde utilizouse do aplicativo servidor de mapas i3Geo, SIG Web livre, criado pelo 
Ministério do Meio Ambiente (MMA), utilizado para gerar o Atlas Digital Ecoturistico do Parque Natural Municipal do Curió (PNMC). O PNMC é uma Unidade de Conservação localizada no município de Paracambi, região metropolitana do Estado do Rio de Janeiro. Este SIG Web, por adotar padrões internacionais de interoperabilidade (OGC - Open Geospatial Consotium - <http://www.opengeospatial.org/>), foi escolhido no intuito de ser uma opção livre e sem os problemas de compra ou renovação de licenças de outros softwares proprietários, além de ser produzido por um órgão governamental, portanto, ideal para a sua utilização em Prefeituras.

\section{SERVIDOR DE MAPAS NA WEB E A SUA IMPORTÂNCIA NA DISSEMINAÇÃo DOS DADOS ESPACIAIS E ACESSO A INFORMAÇÃO GEOGRÁFICA}

Com o advento dos servidores de mapas e da internet, pode-se oferecer aos usuários de dados geográficos o acesso aos dados de forma interativa, dinâmica e gratuita (PARMA, 2007). Os servidores de mapas via internet podem representar a interface entre os usuários e os dados geográficos armazenados em um ou vários servidores de banco de dados, possibilitando a integração com o usuário e a disseminação dos dados geográficos.

No Brasil, os servidores de mapas surgem como importante ferramenta para suprir a demanda que existe atualmente para a disseminação de dados geográficos. A disseminação de dados geográficos brasileiros teve início com a sua instrumentalização através dos Decreto-Lei $n^{\circ} 12.527 / 2011$, de 18 de novembro e Decreto-Lei $n^{\circ}$ 6.666/2008, de 27 de novembro.

Levando em consideração o fato das tecnologias livres serem isentas de royalties, os softwares livres surgem no contexto de servidores web como opções viáveis e em muitos casos até mais vantajosa que as aplicações comerciais. Exemplos de aplicações livres são representados pelos programas AlovMap, Spring Web, Geoserver, Mapserver e i3Geo, cada um deles com determinadas características e peculiaridades para atender a mesma demanda básica de usuários deste tipo de aplicação, que é disseminar dados geográficos na web. Ressalta-se que, as aplicações livres demandam conhecimento técnico muito maior se comparado as aplicações 
comerciais, para disponibilizar os dados geográficos conforme planejado.

Os Web GIS ou SIG Web são mapas dinâmicos e interativos que possuem informações de um banco de dados integradas às informações de elementos gráficos em um único mapa. Permitem também adicionar ou subtrair temas na forma de camadas a serem visualizadas, executar operações de zoom, entre outras funcionalidades, que dependerá de qual software ou aplicativo for utilizado para desenvolver o mesmo. A principal diferença em relação à alternativa dos mapas dinâmicos somente para ver, reside no fato de não ser necessário no SIG Web o usuário instalar no desktop qualquer outro software ou aplicativo adicional especificamente, mesmo de SIG, para poder ter acesso aos mapas. Estes podem ser manipulados diretamente por banco de dados em ambiente cliente-servidor e ou na nuvem (clound).

\section{METODOLOGIA UTILIZADA}

A metodologia desenvolvida na construção do Atlas Digital Ecoturístico para o Parque Natural Municipal do Curió utilizou softwares livres, tanto de SIG como de SIG Web. Foram utilizados os seguintes softwares: o i3Geo (Interface Integrada para Internet de ferramentas de Geoprocessamento) na sua versão 4.6, desenvolvido pelo Ministério do Meio Ambiente do Brasil (disponível para download no Portal do Software Público Brasileiro - <http://www.softwarepublico.org.br>); o Quantum GIS (QGIS) na versão 1.8, que é um sistema de informações geográficas (SIG) open source, licenciado sobre GNU GPL (General Public Licence), sendo um projeto oficial da open source geoespatial foundation (OSGEO, 2012); e o editor de texto livre chamado SciTE.

O i3Geo se destaca em relação a outros softwares correlatos devido a um conjunto de funcionalidades pouco comuns a softwares dessa categoria, nele o usuário final pode alterar a simbologia utilizada na representação dos dados geoespaciais, inserir seus próprios textos e elementos gráficos, algumas opções de análise geográficas são oferecidas, como a geração de buffers, consultas baseadas em tabelas de atributos, entre outras funcionalidades. Além disso, ele supre diversos requisitos 
comumente necessários na implementação de uma IDE (Infraestrutura de Dados Espaciais), como o fornecimento de serviços nos padrões OGC ou download de arquivos (MORRETI, 2011).

O i3Geo possui como interface padrão para a representação espacial que for desejada veicular na Web, mas também possui conjunto de ferramentas que visam complementar a sua interação com outros aplicativos web que trabalham com informações georreferenciadas. Exemplo de ferramenta que foi utilizada no i3Geo para o presente trabalho é o Google Maps. Esta ferramenta possibilita a interação com os usuários e os planos de informação do banco de dados na interface do Google Maps disponibilizada pelo i3Geo. O banco de dados foi disponibilizado em 9 unidades temáticas, totalizando 33 temas (planos de informação) provenientes dos mapeamentos do plano de manejo do PNMC, destacando as trilhas e atrativos do Parque. O projeto seguiu 7 etapas metodológicas, sucintamente descritas na Figura 1.

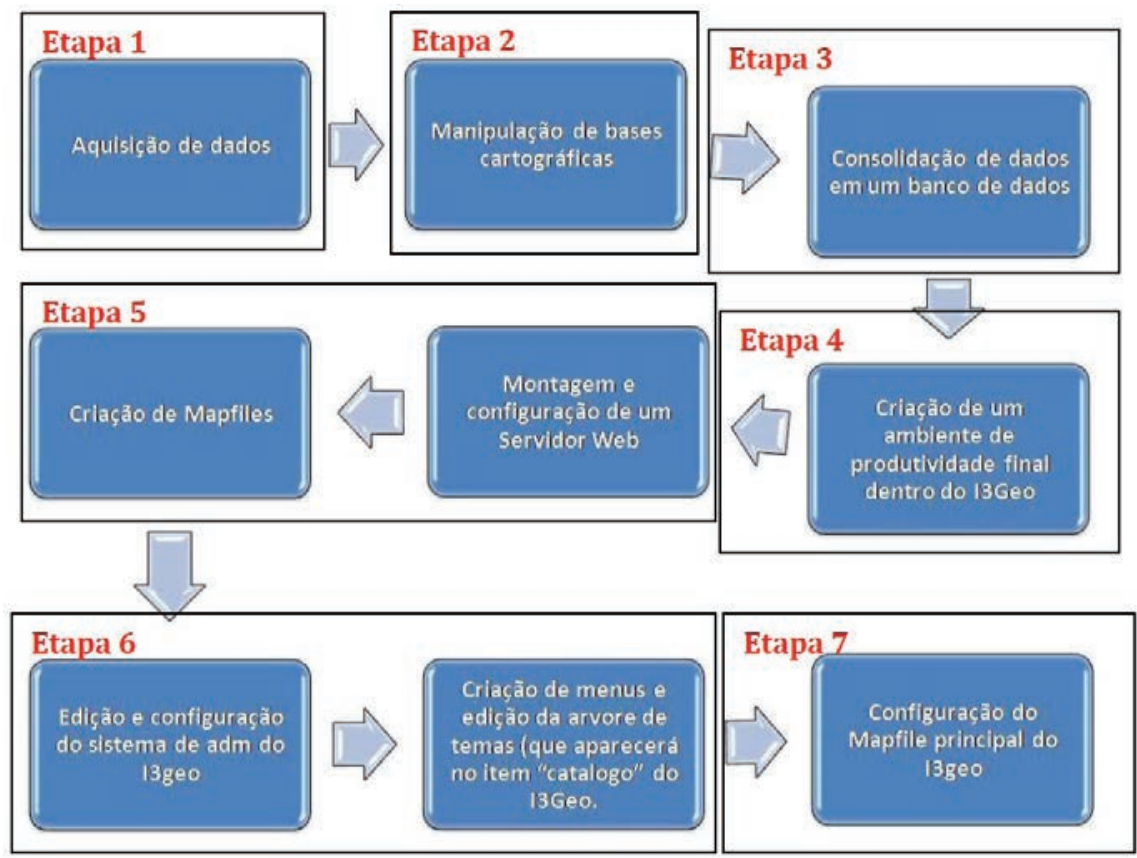

Figura 1 - Etapas metodológicas no i3Geo para confecção do Atlas do PNMC Fonte: Davyd S. de Paiva (2014) 


\section{APRESENTAÇÃo doS DAdOS E DI SUCINTA DOS PRINCIPAIS RESULTAdos}

Com a possibilidade de instalação do i3Geo no ambiente cliente-servidor da Prefeitura de Paracambi, conseguiu-se dessa forma ampliar o acesso aos dados geográficos do parque no site oficial da Prefeitura, em fase de renovação, construindo uma home-page para o PNMC. No link "Atlas" do site, o usuário é remetido ao Atlas Digital no ambiente i3Geo modificado.

O aplicativo i3Geo foi concebido para funcionar em computadores servidores, por isso o uso de testes em ambiente cliente-servidor proporcionado pela SERAD/SR-2/UERJ foi primordial para que o projeto pudesse desenvolver-se plenamente no ambiente cliente-servidor da Prefeitura de Paracambi.

Portanto, os arquivos do Atlas no i3Geo foram repassados para a gestão do parque (SEMADES - Prefeitura Municipal de Paracambi), como um legado positivo deste trabalho. Esses órgãos criaram um endereço de url de hospedagem dos mapas em <http://www.i3Geo.paracambi.rj.gov. br> (Figura 2). O acesso remoto externo ao servidor da prefeitura de Paracambi conseguiu obter uma ótima velocidade de acesso aos dados do Atlas do PNMC, com maior rapidez em relação aos testes do servidor da SERAD/SR-2/UERJ.

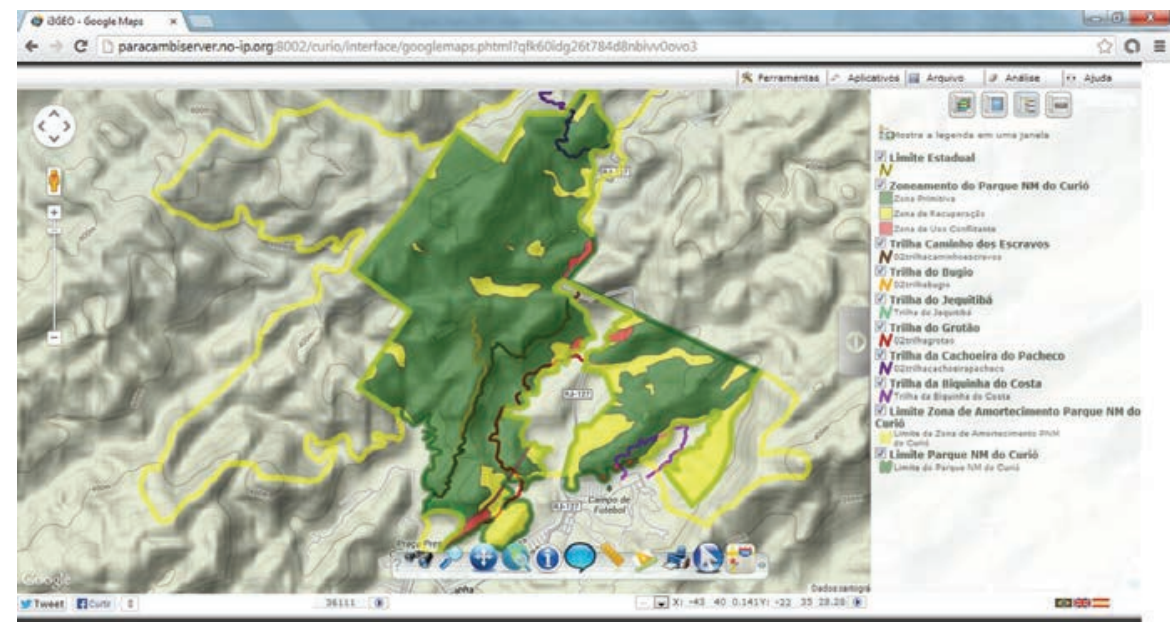

Figura 2 - Atlas do Parque Natural Municipal do Curió (PNMC), constando limites, Zona de Amortecimento, trilhas e mapa do Zoneamento (de acordo com plano de manejo - SEMADES, 2010), no ambiente cliente-servidor disponível no site (acessível diretamente pela url $<$ i3geo.paracambi.gov.rj.br>) 


\section{PRINCIPAIS CONCLUSÕES}

Com a utilização do SIG Web i3Geo foi possível a criação de uma plataforma na internet capaz de divulgar os atrativos ecoturísticos do Parque Natural Municipal do Curió em Paracambi, não só com informações sobre suas trilhas, mas também com informações sobre seus aspectos físicos, contribuindo assim para o desenvolvimento do turismo (e do ecoturismo) no município e disseminando dados geográficos do parque para futuros estudos por pesquisadores e ou técnicos da SEMADES - Prefeitura Municipal de Paracambi. Por sua vez, o i3Geo em sua própria definição, liberdade de uso e funções, permitiu alcançar o pleno objetivo e desenvolvimento do trabalho junto ao apoio dos gestores do PNMC. A mesma liberdade que é o uso de um SIG Web livre é o uso de um GIS Livre como foi o QGIS que por ser de código aberto e possuir interface fácil e interativa, também dispõe atualmente de plugins que possibilitam grande interoperabilidade e ao mesmo tempo propicia interação com o i3Geo.

Outro resultado positivo apresentado neste trabalho foi a de possibilitar o uso de diferentes interfaces no i3Geo, como por exemplo o Google Maps, o que permite uma maior interação entre o usuário do Atlas do Curió que estão mais familiarizados com os dados sendo apresentados em uma visualização amigável e conhecida internacionalmente. Os testes cliente-servidor em ambiente de SIG Web com uma plataforma brasileira que é o i3Geo também possibilitou experienciar um trabalho inédito que ainda não existe entre os órgãos públicos brasileiros que é o de disponibilizar um banco de dados amplo e detalhado (em escalas de 1:10.000) sobre unidades de conservação, principalmente de âmbito municipal.

\section{BIBLIOGRAFIA}

Decreto-Lei Federal $n^{\circ}$ 6.666/2008 de 27 de novembro. Diário Oficial da União. Presidência da República. Brasil. 27 de novembro de 2008.

Decreto-Lei Federal $\mathrm{n}^{\circ}$ 12.527/2011 de 18 de novembro. Diário Oficial da União (Edição Extra). Presidência da República. Brasil. 23 de agosto de 2011.

MORETTI, Edmar (2011) - "Integração do software i3Geo com o gvSIG". In III Jornada Latinoamericana e do Caribe do gvSIG. Foz do Iguaçu, Paraná, Brasil. 
Disponível online no endereço url: http://downloads.gvsig.org/download/ events/jornadas-lac/3as-jornadas-lac/articles/Article-Integracao_i3Geo_gvSIG.pdf (acessado em 15 setembro, 2013).

OSGEO (2012) - "Quantum GIS V.1.8.0 Wroclaw". In Open source geoespatial fondation, Vancouver Disponível online no endereço url: http://qgis.org. Acedido em 15 setembro, 2013.

PARMA, Gabriel Cremona (2007) - Mapas cadastrais na internet: Servidores de mapas. In XIII Simpósio Brasileiro de Sensoriamento Remoto, Florianópolis, Brasil. INPE, p. 1311-1319. Disponível online no endereço url: http://marte. sid.inpe.br/col/dpi.inpe.br/sbsr\%4080/2006/11.16.00.04/doc/1311-1319.pdf. Acendido em 15 setembro, 2013.

SEMADES (2010) - Secretaria Municipal de Meio Ambiente e Desenvolvimento Sustentável. Plano de Manejo do Parque Natural Municipal do Curió. Prefeitura Municipal de Paracambi, Rio de Janeiro. 510 p. 
Série Documentos

Imprensa da Universidade de Coimbra

Coimbra University Press

2015

- U M

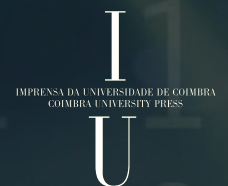

